\title{
Quantitative spectroscopy of single molecule interaction times
}

\author{
H.-H. Boltz, ${ }^{1,2,5}$ (1) A. Sirbu, ${ }^{2}$ N. Stelzer, ${ }^{2}$ M. J. Lohse, ${ }^{2,3}$ C. Schütte, ${ }^{1,4}$ and P. AnNibale ${ }^{2,6}$ \\ ${ }^{1}$ Zuse Institute Berlin (ZIB), 14195 Berlin, Germany \\ ${ }^{2}$ Max Delbrück Center for Molecular Medicine, 13125 Berlin, Germany \\ ${ }^{3}$ University of Würzburg, Institute of Pharmacology and Toxicology, 97078 Würzburg, Germany \\ ${ }^{4}$ Freie Universität Berlin, Institut für Mathematik, 14195 Berlin, Germany \\ ${ }^{5}$ e-mail: boltz@zib.de \\ ${ }^{6}$ e-mail:paolo.annibale@mdc-berlin.de
}

Received 9 November 2020; revised 22 February 2021; accepted 22 February 2021; posted 22 February 2021 (Doc. ID 413030 ); published 19 March 2021

\begin{abstract}
Single molecule fluorescence tracking provides information at nanometer-scale and millisecond-temporal resolution about the dynamics and interaction of individual molecules in a biological environment. While the dynamic behavior of isolated molecules can be characterized well, the quantitative insight is more limited when interactions between two indistinguishable molecules occur. We address this aspect by developing a theoretical foundation for a spectroscopy of interaction times, i.e., the inference of interaction from imaging data. A non-trivial crossover between a power law to an exponential behavior of the distribution of the interaction times is highlighted, together with the dependence of the exponential term upon the microscopic reaction affinity. Our approach is validated with simulated and experimental datasets. () 2021 Optical Society of America under the terms of the OSA Open Access Publishing Agreement
\end{abstract}

https://doi.org/10.1364/OL.413030

Recent progresses in the field of fluorescence single molecule methods [1] have made single molecule tracking (SMT) of membrane receptors in a widefield microscope a technique within reach of many laboratories. This has benefited significantly those researching cell membrane receptor biophysics and pharmacology since these methods offer the opportunity to probe dynamic processes such as oligomerization [2], interaction with downstream signaling partners [3], and trafficking [4], as well as conformational changes at the level of the isolated receptor [5]. Combinations of efficient and photo-stable labeling strategies with advanced optical imaging methods yield spatial-temporal resolutions that allow for nanometer (nm)-level detection [6-8] and millisecond-temporal resolution tracking of individual molecules [9]. Despite the apparent relative simplicity of experimental preparation and data acquisition, data analysis and interpretation remain fraught with significant caveats. In particular, given the continuous nature of the molecular point-spread-function (PSF), defining the duration of a molecular interaction is not trivial: as two identically labeled particles approach each other, their PSFs become unresolvable. While this issue was addressed in static datasets exploiting the notion of stochastic activation and localization [10], for dynamic datasets, where all molecules present are visible at the same time, the problem is still present. The answer to this question will allow us to address the important problem of whether and how these particles are (constructively) interacting, revealing details on microscopic interaction rates. This ultimately pertains to data interpretation and, therefore, is conceptually independent of the experimental detection quality achieved.

Previous work $[2,3,11,12]$ has addressed this issue by generating and evaluating a histogram (or distribution) of molecular overlap (or colocalization) times. As the identification of colocalized particles is fundamentally easier, if they are labeled differently, we will only address the (more complex, but also more typical) monochromatic case. Furthermore, we are limited to a density regime, where trimers and higher order clusters are irrelevant and particles are found as either monomers or dimers.

We begin by considering a related problem that can be handled analytically: the distribution $p_{\mathrm{fp}}$ of first-passage times of a freely diffusing particle through a circular boundary. We focus on standard Brownian diffusion, but extension to anomalous diffusion is possible and may be relevant [13]. This serves as a continuum reference theory to understand the overall shape and dependencies of the overlap time distribution. We denote the particle's diffusion constant by $D$ and the radius of the circle (approximating the PSF) by $R$. Dimensionally, the only time scale in the problem is $\tau=R^{2} / D$. Hence, one expects an asymptotically exponential large time tail. For very small times, the particle is blind to the scale of the region, and one expects [14] a scale-free distribution. This can be summarized as

$$
p_{\mathrm{fp}}(t) \sim \begin{cases}t^{-3 / 2} & \text { for } 0<t \ll \tau \\ \exp \left(-t / \tau_{\mathrm{fp}}\right) & \text { for } t \gg \tau \text { with } \tau_{\mathrm{fp}} \approx \tau / 5.78\end{cases}
$$

Details of this calculation have been discussed earlier $[15,16]$ and are also provided in Supplement 1. Similar findings have been made using approaches with explicit model interaction potentials [17]. Graphically, these two behaviors can be interpreted as two "classes" of trajectories, as summarized in Fig. 1: short trajectories (purple) that only explore the rim and long trajectories (blue) that traverse the whole colocalization area/PSF. 

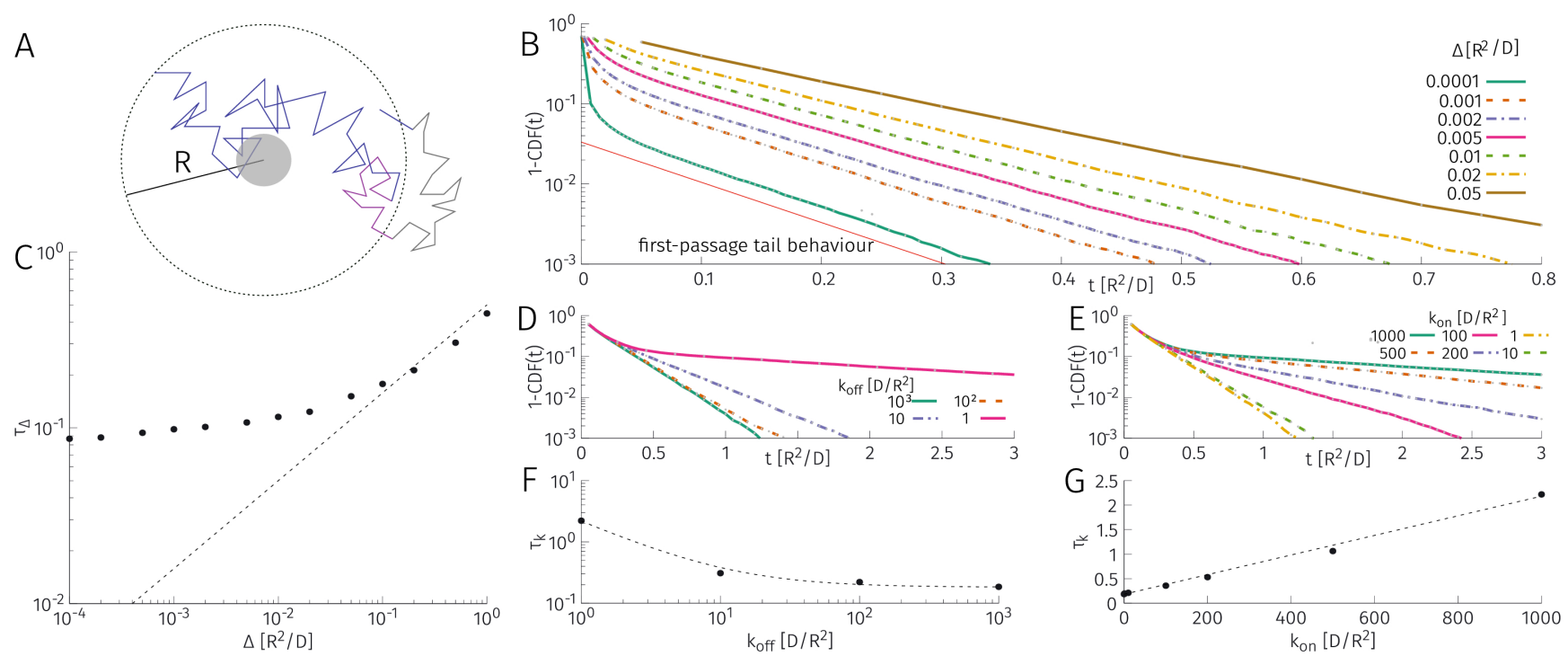

Fig. 1. A, simulation setup highlighting the difference between the first-passage time and the colocalization time at finite acquisition time $\Delta$. For some $\Delta$, the particle will be considered to never have passed, even though the parts (gray) of the trajectory are outside. B, distribution of colocalization times generated computationally for various $\Delta$. When the temporal resolution is adequate, the distribution of colocalization times has two functional components: a rapid power law and a slower exponential decay. C, numerically recovered time scale from the exponential part. The straight line corresponds to $\tau_{k} \sim \sqrt{\Delta}$ as a guide to the eye. $\mathrm{D}$, dependence of the distribution of colocalization times as a function of $k_{\mathrm{off}}$ and, $\mathrm{E}$, as a function of the $k_{\mathrm{on}}$. Both use $\Delta=0.05 R^{2} / D$. The dependence of the resulting slope of the exponential component of the colocalization time distributions in $\mathrm{D}$ and $\mathrm{E}$ is displayed, respectively, in $\mathrm{F}$ and $\mathrm{G}$, highlighting the $\tau_{k} \sim k_{\mathrm{off}}^{-1}+$ const and $\tau_{k} \sim k_{\mathrm{on}}+$ const behavior, respectively.

As we will show below, the overall description provided by Eq. (1) holds quite generally.

Experimental observation is stroboscopic: images are taken at a finite acquisition rate. Thus, a trajectory that ventures outside the region of interest, cp. the gray segment in Fig. 1A, may be considered within the region at every time of observation. The actual problem is to determine the first-observed passage when considering snapshots (the effective PSF has contributions from imaging as well as motion blur and finite detection precision; this is discussed in Supplement 1) taken at a fixed acquisition time interval $\Delta$. Thus, we need to determine how the distribution $p_{\Delta}$ inferred from the discrete acquisition will deviate from the continuous first-passage analysis presented in the previous paragraph. We shall note here that the dimensional arguments still hold, since the first-passage tail time scale $\tau_{\mathrm{fp}}$ is a lower bound to the observed time scale $\tau_{\Delta}$ with $p_{\Delta}(\tau) \sim \exp \left(-t / \tau_{\Delta}\right)$, i.e., $\tau_{\Delta} \geq \tau_{\mathrm{fp}}$, as the discrete observation can only extend the apparent colocalization time. Analogously, we expect monotony and, ultimately, $\tau(\Delta) \sim \sqrt{\Delta}$ as the probability within the colocalization region becomes uniformly spread for large times and, thus, the probability to be observed outside grows with $\Delta$ as the size of the zone at the rim that can reach the outside with one step. We validated this intuitive argument by numerical simulations of diffusing particles using direct integration of the Langevin equation, $d x_{i}^{n} / d t=\sqrt{2 D} \eta(t)$, with $i=x, y$ indexing spatial dimensions, $n=1 \ldots N$ indexing particles, and $\eta(t)$ denoting a Gaussian random force with $\langle\eta(t)\rangle=0$ and $\left\langle\eta(t) \eta\left(t^{\prime}\right)\right\rangle=\delta\left(t-t^{\prime}\right)$. The particles are considered colocalized if their distance is not larger than $R$, as depicted in Fig. 1B. We show empirical cumulative distributions, $\operatorname{CDF}(t)=1 / N_{t} \sum_{i}^{N_{t}} \Theta\left(t-t_{i}\right)$; intuitively one can think of $1-\operatorname{CDF}(t)$ as the fraction of colocalization events lasting longer than $t$. Figure 1B illustrates the time spent inside the circle as a function of the acquisition time. If the acquisition time is adequate, we observe the expected combination of a rapid power law decay and a slower exponential behavior of the distribution. The slope of the exponential part will reflect the presence of an interaction between the particles, if any. Interestingly, Fig. 1C highlights how the recovered time scale of the exponential tail depends on the acquisition time. As the time $\Delta$ grows higher, the time scale increases, suggesting "apparent interactions" also in this negative control. This increase is in line with our expectation $\tau(\Delta) \sim \sqrt{\Delta}$ and stresses the importance of a careful examination of the behavior of freely diffusing particles under actual experimental conditions.

We then extended this approach to interacting molecules using a Doi-like model [20] in which the interaction is described by three parameters: a binding rate $k_{\text {on }}$, an unbinding rate $k_{\text {off }}$, and an interaction range $\ell$. Pairs of molecules $i, j$ whose distance is within the range, $r_{i j}<\ell$, bind with constant rate $k_{\text {on }}$. Rather than introducing an explicit new construct formed this way, we let bound particles continue to diffuse while enforcing a constraint $r_{i j}<\ell$, corresponding to soft molecules. This is justified by noticing that our point particle description neglects most of the internal degrees of freedom. From the bound state, molecule pairs unbind with constant rate $k_{\text {off }}$, after which they can leave each other's vicinity again. Previously, the tail behavior of the overlap time distribution (or the excess time compared to a freely diffusing background) has been identified with $k_{\text {off }}^{-1}$. Intuitively, the typical time spent in the bound state should also depend on the binding rate and the range, as those control the probability to be bound and, thus, the number of unbinding events, each of which takes a time $\sim k_{\text {off }}^{-1}$. We recover the expected dependence on $k_{\text {off }}$, as illustrated in Fig. 1D, i.e., the higher $k_{\text {off }}$, the shorter the lifetime of the interaction. Interestingly, however, also the $k_{\text {on }}$ plays a role, indicating the 
importance of recaptures on the observed distribution of interaction times, as shown in Fig. 1E. If we look at the dependence of the recovered lifetime of the interaction $\tau_{k}($ Fig. $1 \mathrm{~F})$, we observe that, at very high $k_{\text {off }}$, there is a residual lifetime that agrees with the result for a freely diffusing monomeric particle that is observed also in the negative control (Fig. 1A). Furthermore, as the interaction becomes stronger (smaller $k_{\text {off }}$ ), the recovered lifetime scales linearly with $k_{\text {off }}^{-1}$ and $k_{\text {on }}$ (see Figs. $1 \mathrm{~F}$ and $1 \mathrm{G}$ ). We can formalize this intuition (see Supplement 1 ) by calculating the typical time spent within the colocalization region as a function of the affinity $K=k_{\text {on }} k_{\text {off }}^{-1}$ as

$$
\tau(K)=\tau(0)+\text { const } \cdot K \text {. }
$$

The proportionality factor depends on the size of the interaction region. We conclude that introducing an interaction adds a term scaling as $k_{\text {off }}^{-1}$ to the time scale $\tau(0)=\tau_{\Delta}$ observed in the exponential tail, but one cannot identify these with each other, in particular there is a dependence on the binding rate $k_{\text {on }}$. This dependence is relevant in comparative studies where the observed time scales might be used to assess the difference in unbinding rate as a proxy for different dimerization behavior. Furthermore, the overall gestalt of the overlap time distribution is basically unaltered, i.e., we see the same algebraic initial behavior crossing over into an exponential tail with a time scale that follows Eq. (2). The reasoning behind this is that the practically relevant regime is $\ell \ll R$, i.e., the interaction range is small compared to the colocalization range. Thus, the scale-free behavior, formed by trajectories that explore a small region close to the edge of the colocalization range, will be unaffected by the interaction. Within the graphical approach used earlier (Fig. 1A), it is rather intuitive that the overall shape of the overlap time distribution remains unchanged: short trajectories on the rim (purple) corresponding to the algebraic beginning remain unchanged, long trajectories (blue) will eventually hit the interaction region and therefore get extended.
As a byproduct, the non-exponential behavior for short times gets more pronounced when the excess time due to interaction is large. For practical purposes, typical order of magnitudes for the kind of membrane receptors we are considering are $R \approx 300 \mathrm{~nm}, D \approx 0.1(\mu \mathrm{m})^{2} / s$, implying a typical time scale of $\tau=R^{2} / D<1$ s. The length scale $R$ as well as the time scale $\tau$ are large compared to typical interaction scales of up to tens of $\mathrm{nm}$ and seconds for strongly interacting molecules. This highlights the great importance of carefully evaluating and subtracting background diffusion.

We then moved to performing actual SMT on simulated datasets, mimicking real experimental conditions. We simulated movies (see Visualization 1 for an exemplary movie) of $10^{4}$ frames, containing $10^{2}$ particles each, with an acquisition time of $\Delta=0.05 R^{2} / D$. Figure 2 illustrates the results of the $\mathrm{u}$-track package [21], where the interaction between the particles is arbitrarily modulated. Figures $2 \mathrm{~A}-2 \mathrm{D}$ display the distribution of colocalization times detected by the software on the simulated dataset (green), the ground truth (i.e., the true distribution of colocalization times from the simulated particle positions), and the fit to the detected data in the exponential tail. We generated the ground truth by employing a simple thresholding procedure that identifies every pair with a distance below $R_{c} \approx 1.5 R$ as colocalized (see Supplement 1). The detected distribution of colocalization times matches the "true" values for the noninteracting sample (Fig. 2A), and samples displaying increasing interaction strength (Figs. 2B-2D). Once the apparent $k_{\text {off }}$ (from the fit to the distribution of detected interaction times) is plotted against the simulated $k_{\text {off }}$, Fig. $2 \mathrm{E}$ illustrates that the detected and true values are in excellent agreement. The offset due to the random interaction is clearly visible in the data. When the random colocalization offset is subtracted, the recovered $k_{\text {off }}$ correlates with the simulated one, as displayed in Fig. 2F.

We then moved to apply this analysis framework to experimental datasets. We chose to analyze the behavior of the two
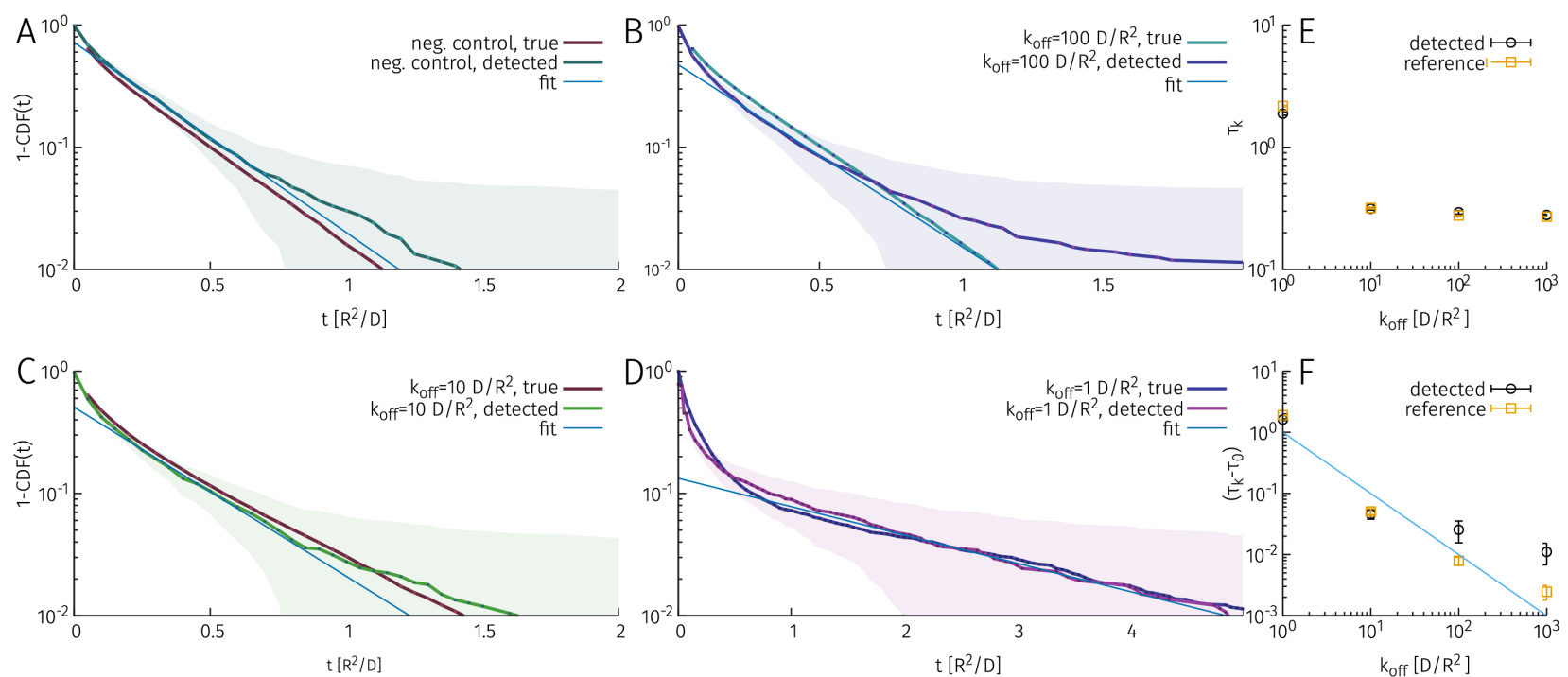

Fig. 2. Distribution of colocalization times obtained running a commercial single-particle tracking software on simulated datasets. A-D, recovered distribution of colocalization times for a non-interacting control. Displayed are the true interaction values (known from the simulated centers of each molecule at any given time) and the output of the tracking software (detected), as indicated in the legend. The slope of the exponential component of the detected colocalization time distribution is fit to extract the apparent lifetime of the interaction $\tau$. Shaded bands highlight $95 \%$ confidence level for the detected distribution $[18,19]$. Fits were performed with gnuplot in the region for which $1-\operatorname{CDF}(t) \in[0.05: 0.3]$. E and F, recovered apparent lifetimes as a function of the interaction strength $\left(k_{\text {off }}\right)$. In $\mathrm{F}$, the background contribution is subtracted. 

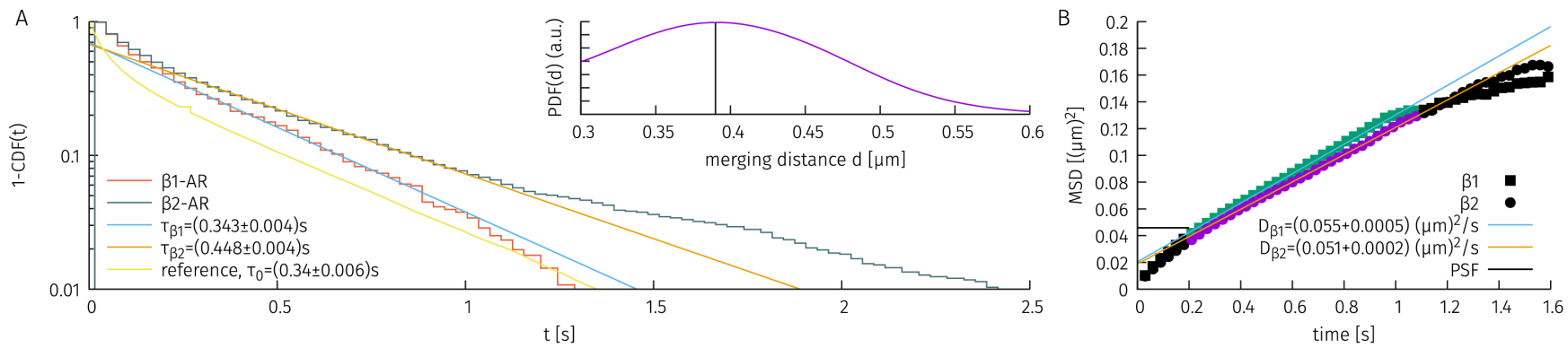

Fig. 3. Analysis of inter-molecular interaction for the SNAP- $\beta_{1}-\mathrm{AR}$ and SNAP- $\beta_{2}$-AR GPCRs in CHO cells labeled with SnapSurface 549 . A, distribution of overlap times for the two $\beta$-AR. Inset, empirical PDF (using Kernel density estimation) of distances of merging particles (larger than the PSF) in the frame before merging. B, mean-square displacement indicating no trivial difference in diffusivities as the origin of the different overlap behavior. The inferred values of diffusion constant and threshold distances were used to obtain a suitable reference.

well-characterized $G$ protein-coupled receptors (GPCRs) $\beta_{1}$ - adrenergic (AR) and $\beta_{2}$-AR [2,22,23]. Figure 3 illustrates the result of our analysis of single-particle TIRF movies of $N$-terminally SNAP-tagged (a fusion construct allowing extracellular labeling with a bright organic fluorophore of choice; see Supplement 1 for more experimental details) $\beta_{1}$-AR and $\beta_{2}$-AR diffusing on the basolateral membrane of $\mathrm{CHO}$ cells. Figure $3 \mathrm{~A}$ displays the detected distribution of colocalization times, together with the distribution from a randomly diffusing control. The higher dimer lifetime of the $\beta_{2}-\mathrm{AR}$ is in line with previous observations from our group of a higher steady-state dimerization for this receptor [2]. The lifetime of the $\beta_{1}$-AR is close to the reference of the non-interacting, reference control. The diffusivities in Fig. 3B indicate that the difference in overlap time is not due to a trivial difference in the free dynamics.

In this Letter, we addressed to what extent a characterization of inter-molecular interactions is possible by means of single molecule imaging with a focus on fundamental interpretation issues rather than technical aspects of detection or tracking. We have derived the shape of the distribution of overlap times both with and without an interaction providing a solid theoretical foundation to this important methodology. This analysis reveals that there is a non-exponential, algebraic behavior for small overlap times and an exponential tail for large times, whose time scale depends on the acquisition time. For interacting molecules, we find that the quantity accessible from the exponential tail is the affinity, i.e., the ratio of binding and unbinding rates. This would only allow quantitative comparisons between molecules imaged under the same experimental conditions and poses an intriguing challenge for future work to extract the individual rates. These determinations were confirmed when using a common software on simulated datasets. We finally applied our framework to address the difference in dimerization kinetics between two GPCRs, the $\beta_{1}$ - and $\beta_{2}$-adrenergic receptors.

Funding. Deutsche Forschungsgemeinschaft (421152132 (subproject C03)), EXC 2046 (MATH+Incubator project IN-B2).

Disclosures. The authors declare no conflicts of interest.

Supplemental document. See Supplement 1 for supporting content.

\section{REFERENCES}

1. T. Schmidt, G. Schütz, W. Baumgartner, H. Gruber, and H. Schindler, Proc. Natl. Acad. Sci. USA 93, 2926 (1996).
2. D. Calebiro, F. Rieken, J. Wagner, T. Sungkaworn, U. Zabel, A. Borzi, E. Cocucci, A. Zürn, and M. J. Lohse, Proc. Natl. Acad. Sci. USA 110, 743 (2013).

3. T. Sungkaworn, M.-L. Jobin, K. Burnecki, A. Weron, M. J. Lohse, and D. Calebiro, Nature 550, 543 (2017).

4. Z. Y. Weinberg and M. A. Puthenveedu, Traffic 20, 121 (2019).

5. G. G. Gregorio, M. Masureel, D. Hilger, D. S. Terry, M. Juette, H. Zhao, Z. Zhou, J. M. Perez-Aguilar, M. Hauge, S. Mathiasen, J. A. Javitch, H. Weinstein, B. K. Kobilka, and S. C. Blanchard, Nature 547, 68 (2017).

6. K. I. Mortensen, L. S. Churchman, J. A. Spudich, and H. Flyvbjerg, Nat. Methods 7, 377 (2010).

7. N. Chenouard, I. Smal, F. De Chaumont, M. Maška, I. F. Sbalzarini, Y. Gong, J. Cardinale, C. Carthel, S. Coraluppi, M. Winter, A. R. Cohen, W. J. Godinez, K. Rohr, Y. Kalaidzidis, L. Liang, J. Duncan, H. Shen, Y. Xu, K. E. G. Magnusson, J. Jaldén, H. M. Blau, P. Paul-Gilloteaux, P. Roudot, C. Kervrann, F. Waharte, J.-Y. Tinevez, S. L. Shorte, J. Willemse, K. Celler, G. P. van Wezel, H.-W. Dan, Y.-S. Tsai, C. O. de Solórzano, J.-C. Olivo-Marin, and E. Meijering, Nat. Methods 11, 281 (2014).

8. C. Manzo and M. F. Garcia-Parajo, Rep. Prog. Phys. 78, 124601 (2015).

9. H. Shen, L. J. Tauzin, R. Baiyasi, W. Wang, N. Moringo, B. Shuang, and C. F. Landes, Chem. Rev. 117, 7331 (2017).

10. E. Betzig, G. H. Patterson, R. Sougrat, O. W. Lindwasser, S. Olenych, J. S. Bonifacino, M. W. Davidson, J. Lippincott-Schwartz, and H. F. Hess, Science 313, 1642 (2006).

11. R. S. Kasai and A. Kusumi, Curr. Opinion Cell Biol. 27, 78 (2014).

12. J. Möller, A. Isbilir, T. Sungkaworn, B. Osberg, C. Karathanasis, V. Sunkara, E. O. Grushevskyi, A. Bock, P. Annibale, M. Heilemann, C. Schütte, and M. J. Lohse, Nat. Chem. Biol. 16, 946 (2020).

13. R. Metzler, J.-H. Jeon, A. G. Cherstvy, and E. Barkai, Phys. Chem. Chem. Phys. 16, 24128 (2014).

14. M. E. Fisher, J. Stat. Phys. 34, 667 (1984).

15. E. N. Govorun, V. A. Ivanov, A. R. Khokhlov, P. G. Khalatur, A. L. Borovinsky, and A. Y. Grosberg, Phys. Rev. E 64, 040903 (2001).

16. G. Oshanin, M. Tamm, and O. Vasilyev, J. Chem. Phys. 132, 235101 (2010).

17. G. Margolin and E. Barkai, Phys. Rev. E 72, 025101 (2005).

18. P. Massart, Ann. Probab. 18, 1269 (1990).

19. A. Dvoretzky, J. Kiefer, and J. Wolfowitz, Ann. Math. Stat. 27, 642 (1956).

20. M. Doi, J. Phys. A 9, 1479 (1976).

21. K. Jaqaman, D. Loerke, M. Mettlen, H. Kuwata, S. Grinstein, S. L. Schmid, and G. Danuser, Nat. Methods 5, 695 (2008).

22. H. P. Valentine, Mol. Biol. Cell 22, 2970 (2011).

23. P. Annibale and M. J. Lohse, Nat. Methods 17, 273 (2020). 